\title{
Enseñar física con Juego de Tronos: Estudio del movimiento
}

\author{
A. J. Franco-Mariscal \\ Didáctica de las Ciencias Experimentales, \\ Universidad de Málaga. Málaga (Spain). \\ e-mail:anjoa@uma.es
}

Received 7 May 2020; accepted 30 July 2020

\begin{abstract}
Este trabajo presenta algunos ejemplos de cómo llevar al aula de física el estudio de la cinemática a través del análisis de diferentes escenas de la serie de TV Juego de Tronos, las cuales se pueden explicar a partir de los conceptos de esta materia. En primer lugar, se proponen situaciones para introducir los conceptos de sistema de referencia y movimiento, y para diferenciar entre trayectoria, espacio recorrido y desplazamiento. Luego, se abordan posición, velocidad y aceleración como magnitudes fundamentales del movimiento. Se finaliza con el planteamiento de problemas abiertos de los distintos tipos de movimientos rectilíneos y circulares.
\end{abstract}

Descriptores: Física en series de TV; Juego de Tronos; cinemática.

This paper presents some examples of how to study kinematics in the Physics classroom through the analysis of different scenes from the TV Series Game of Thrones, which can be explained using concepts of the subject. First, situations are proposed to introduce the concepts of reference system and motion and to differentiate between trajectory, distance, and displacement. Then, position, velocity, and acceleration are addressed as fundamental magnitudes of motion. It ends with some open problems solving of the different types of rectilinear and circular motions.

Keywords: Physics in TV series; Game of Thrones; kinematics

DOI: https://doi.org/10.31349/RevMexFisE.18.63

\section{Las series de TV como recurso educativo para enseñar física}

El interés del alumnado hacia las ciencias ha sido una preocupación importante en el panorama español [1], puesto que existe una valoración negativa de la física por parte de los estudiantes de secundaria, quienes la perciben como una asignatura excesivamente difícil y aburrida, alejada de su vida cotidiana, con pocas posibilidades de éxito y sin futuro profesional [2].

Para superar este obstáculo, algunos autores han propuesto diferentes estrategias para motivar y mejorar el aprendizaje y la actitud del alumnado hacia esta materia. Una de ellas consiste en el uso de los medios de comunicación como fuente de aprendizaje; y en concreto, de la televisión, el medio más consumido en España en 2018 [3], y que se sitúa junto con Internet como los más extendidos entre la población adolescente. Los datos de consumo de televisión indican que las series de ficción son las preferidas entre los jóvenes, a la vez que ponen de manifiesto su influencia en las concepciones de los estudiantes y en la imagen que se presenta de la ciencia en general, y de la física en particular, la cual no suele coincidir con la realidad [4]. Es importante resaltar que el uso de documentos de ficción en general tiene una tradición que se remonta a Isaac Asimov.

Diferentes estudios han explorado el uso de la televisión para enseñar física con buenos resultados en el aprendizaje de estudiantes de secundaria y también en su motivación por aprender esta materia. Algunos ejemplos son el uso de la serie de animación Los Simpsons [5] para identificar y discutir fenómenos de la física, Peppa Pig [6] para aprender óptica o de la ficción Prison Break [7] para estudiar las fuerzas. A pesar de su potencialidad, el número de propuestas e investigaciones realizadas para educar respecto a un buen uso de televisión, y más en particular, para enseñar y aprender física o para crear actitudes positivas hacia ella se considera todavía insuficiente.

\section{Objetivo e hipótesis}

El objetivo de este artículo es presentar una secuencia de sugerencias didácticas para el estudio del movimiento empleando como recurso la serie Juego de Tronos. La hipótesis sigiere que el análisis físico de secuencias bien seleccionadas de la serie Juego de Tronos puede ayudar al alumnado a entender mejor los conceptos relacionados con el movimiento y motivar a estudiantes poco interesados en la física.

\section{Juego de Tronos para enseñar fśica}

Juego de Tronos es una serie de televisión de drama y fantasía, creada por Benioff y Weiss y producida por la cadena HBO, emitida entre 2011 y 2019, y que obtuvo un gran éxito de audiencia a nivel mundial. Se trata de una adaptación de las novelas pertenecientes a la saga Canción de fuego y hielo de G.R. Martin. La serie relata las vivencias de varias familias nobles en el continente ficticio de Poniente para tener el control del Trono de Hierro y gobernar los siete reinos. Medios como The Washington Post, Time o The Guardian [8] la han catalogado como una de las mejores series de ficción y ha recibido numerosos premios Emmy.

La serie ha sido también fuente de inspiración para diferentes estudios que abarcan desde la política [9] hasta la ciencia. En este último ámbito destaca el trabajo de Thompson [10], que explora la ciencia de este mundo fantástico desde 
la climatología, la astronomía, la química o la biología. En el caso de la física, destaca la explicación del muro de hielo.

El presente trabajo aporta como novedad la manera de utilizar algunas escenas de esta serie para el estudio de los conceptos básicos de cinemática, concretamente, de las características del movimiento y los distintos tipos de movimientos, todos ellos incluidos en el currículo de física de educación secundaria y del primer año de universidad.

\section{El movimiento en Juego de Tronos}

\subsection{Movimiento y sistema de referencia}

La serie ofrece muchas oportunidades que permiten introducir el concepto de movimiento como cambio de posición de un cuerpo a lo largo del tiempo: jinetes a caballo, viajes en barco, dragones volando, etc. Se debe indicar que el movimiento depende del sistema de referencia elegido y para ello se puede proponer al estudiante que describa un mismo movimiento, tal como la caída del collar de Daenerys cuando vuela en línea recta sobre su dragón, desde dos observadores distintos. En este caso, un observador en tierra vería el movimiento del collar como curvilíneo, mientras que la propia Reina de Dragones lo vería rectilíneo, debido al sistema de referencia desde donde se observa el movimiento.

\subsection{Trayectoria, espacio recorrido y desplazamiento}

El vuelo del dragón también se puede emplear para estudiar el concepto de trayectoria como la línea descrita por un móvil en su movimiento, indicando que podría ser recta o con distintos tipos de curvas (circular, elipse, parábola, hipérbole, etc.).

Los distintos viajes que se realizan a lo largo de la historia (John Snow desde Invernalia hasta el Muro, el viaje de Daenerys hasta llegar al Trono de Hierro o el trayecto de los caminantes blancos) son útiles para trazar diferentes trayectorias sobre el mapa de los siete reinos y discutir si son rectilíneas o curvilíneas. A continuación se utiliza como ejemplo el viaje de Daenerys desde Pentos hasta Desembarco del Rey (Fig. 1) para explicar cómo se aborda el uso de mapas en el aula, así como el espacio recorrido y desplazamiento.

A partir del mapa, primero se trabajan las escalas. Se expone que una escala indica el número de veces que se ha reducido un espacio o un objeto para representarlo, y que existen dos tipos de escalas; numérica y gráfica. En este caso (Fig. 1), la escala numérica es 1:10.000.000 y significa que $1 \mathrm{~cm}$ del mapa corresponde con 10,000,000 cm $(100 \mathrm{Km})$ en la realidad. En la escala gráfica, la primera sección de color blanco, proporcionada a los estudiantes con la medida de $1 \mathrm{~cm}$, corresponde con $100 \mathrm{Km}$. Asimismo, se indica que una escala permite conocer la distancia real entre dos puntos y se

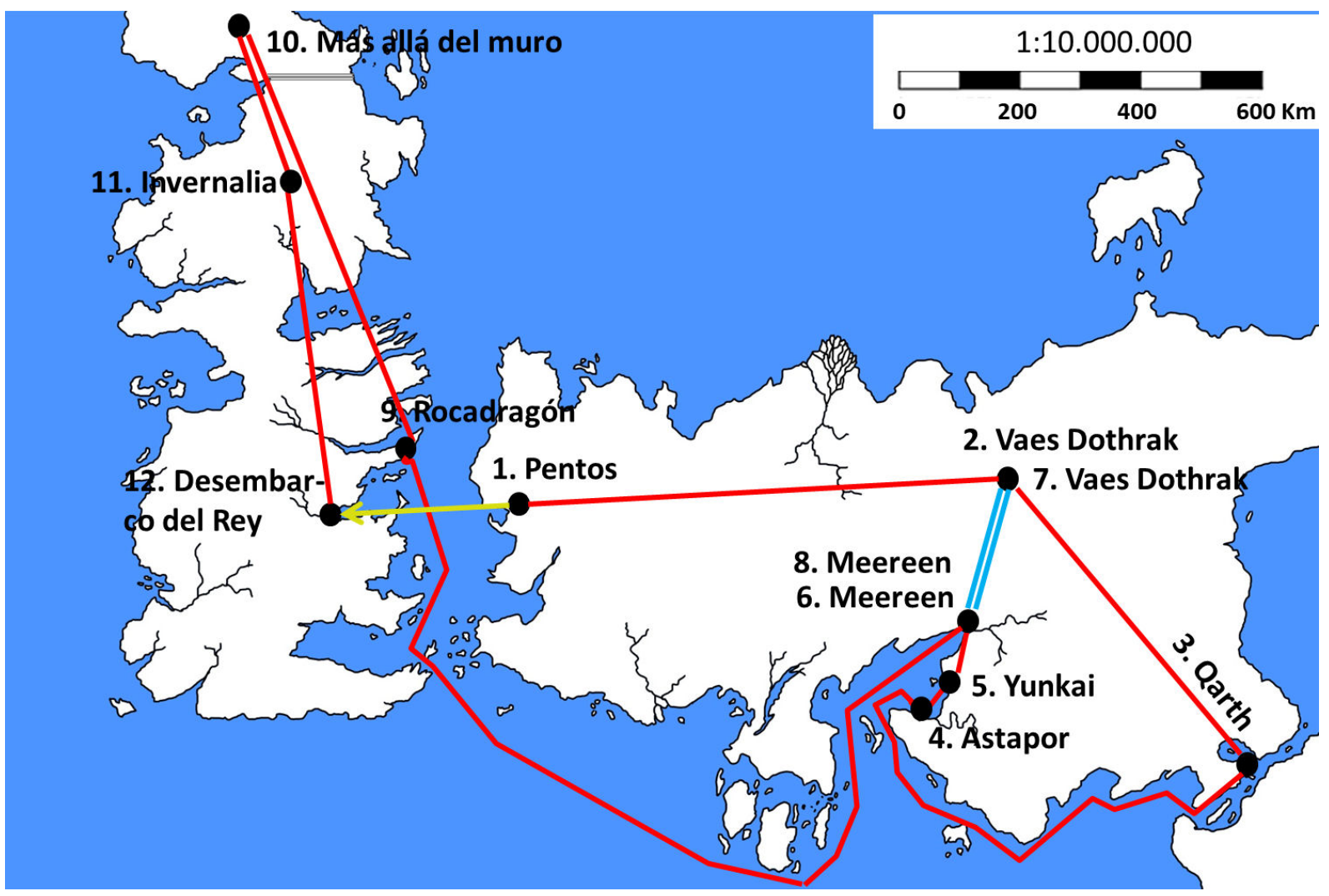

FIGURA 1. Mapa de los siete reinos [elaboración propia]. 
pide calcular la distancia que recorre Daenerys desde Meerin hasta Rocadragón por mar empleando las dos escalas. Para la escala numérica, los estudiantes deben medir con una regla y por tramos, la distancia entre las dos ciudades. Una vez conocida, deben aplicar la razón de proporcionalidad para calcular la distancia real. Para la escala gráfica, primero deben averiguar su significado midiendo con una regla la longitud de la escala y observar la distancia que representa. Luego, deben medir la distancia entre ambas ciudades y aplicar la razón de proporcionalidad longitud real/longitud de la escala. Otra actividad interesante es reproducir una parte del mapa a otra escala.

Se indicará que las medidas realizadas corresponden en física con una magnitud escalar denominada espacio recorrido. A continuación, se introduce el desplazamiento como magnitud vectorial que solo depende de la posición inicial y final en línea recta, y es independiente de la trayectoria. Se pide calcular ambos para el viaje completo de Daenerys y establecer las diferencias. Para el espacio recorrido, los estudiantes deben medir cada una de las distancias entre las ciudades ( 1 a 2,2 a $3, \ldots 11$ a 12 ) y para el desplazamiento, la distancia en línea recta entre 1 y 12, representada en la Fig. 1 en amarillo como un vector que parte de Pentos a Desembarco del Rey.

\subsection{Posición, velocidad y aceleración}

La trayectoria dibujada sobre el mapa se puede utilizar también para definir la posición de un móvil en diferentes puntos como magnitud vectorial y para trabajar mediante el cambio de posición las magnitudes velocidad y aceleración.

Para abordar el concepto de velocidad se puede pedir al estudiante que dibuje un posible recorrido entre dos puntos tales como el Muro y Desembarco del Rey, pasando por diferentes lugares del reino, y que indique el tiempo que ha tardado en recorrer cada tramo. Luego, debe calcular la distancia total y la existente entre los diferentes lugares, usando la escala del mapa y finalmente, la velocidad media en cada tramo y en el recorrido completo para decidir si el viaje se realizó a una velocidad constante o variable. También tiene interés construir y discutir las gráficas espacio-tiempo y velocidad-tiempo del recorrido. Es deseable incluir en el viaje un tiempo de descanso en un lugar para poder discutir cómo sería dicho tramo (horizontal) en la gráfica espaciotiempo.

Por otra parte, a partir de diferentes datos de velocidad tomados en varios puntos del recorrido a ciertos tiempos, se podría averiguar si la aceleración del viajero fue constante o variable.

\subsection{Movimiento rectilíneo y uniforme (MRU)}

El recorrido anterior se puede emplear para abordar el movimiento más sencillo, donde un móvil se desplaza con una trayectoria recta y velocidad constante, denominado movimiento rectilíneo y uniforme (MRU). Así, se podría cal- cular la velocidad media a la que deberíamos viajar a caballo para llegar al destino por el camino más corto en un tiempo dado, o el tiempo que se tardaría en llegar por mar desde un lugar a otro viajando en línea recta y a una velocidad constante conocida.

Además, la serie plantea numerosas batallas campales entre ejércitos: la batalla de los bastardos, Dothraki contra Lannister o la batalla de Invernalia de la larga noche con los caminantes blancos son algunos ejemplos. Estas batallas permiten plantear problemas de encuentro de dos móviles. Se debe comenzar discutiendo el tipo de movimiento de los ejércitos y sus trayectorias. Al tratarse del encuentro de dos móviles enfrentados, lo más lógico es suponer una trayectoria rectilínea y empezar con un caso simple, como un movimiento a velocidad constante. Es interesante que el estudiante proponga datos de espacio y velocidad y que, a partir de ellos, calcule el tiempo que tardan los ejércitos en encontrarse y el espacio donde se cruzan. Así también, el estudiante puede representar la gráfica espacio-tiempo. Un problema tipo podría ser el siguiente:

- El ejército de los Dothraki avanza a caballo en línea recta y a velocidad constante de $60 \mathrm{Km} / \mathrm{h}$ hacia el ejército de los Lannister, que corre a su encuentro a una velocidad constante de $22 \mathrm{Km} / \mathrm{h}$. Si ambos ejércitos están separados $3 \mathrm{Km}$, ¿en qué punto comenzará la batalla y cuánto tiempo tardarán en encontrarse? Representar la gráfica espacio-tiempo de los dos móviles.

\section{Movimiento rectilíneo uniformemente acel- erado (MRUA)}

Un siguiente paso sería introducir movimientos acelerados, donde un móvil va aumentando su velocidad con el tiempo. En definitiva, debemos dirigir al alumnado hacia planteamientos con las características de un movimiento rectilíneo uniforme con aceleración constante (MRUA). Tal caso sería el ataque de Jaime Lannister cabalgando en línea recta hacia el dragón herido y en reposo, lo que permitiría calcular, entre otras cosas, el tiempo que tarda en atacarlo o la aceleración constante a la que debe marchar, a partir de otros datos conocidos.

Luego se podría pasar al movimiento de dos móviles; con ello, se pueden plantear problemas abiertos de persecución y de encuentro. En el primer caso, se puede analizar la escena que tiene lugar en el primer vuelo de Jonh Snow en dragón intentando alcanzar a Daenerys con su dragón. Al suponer esta persecución con MRUA y dados valores para velocidad inicial y aceleración de los dos dragones, el estudiante podría calcular cuánto tiempo tardaría Jonh en alcanzar a Daenerys y en qué punto se encontrarían tomando como referencia el lugar de salida.

Asimismo, se pueden proponer problemas abiertos similares al descrito para la batalla entre Dothraki y Lannister para calcular el punto de encuentro y el tiempo, pero en este 
caso, con ambos móviles desplazándose con aceleración constante, o bien uno de ellos con velocidad constante y el otro con aceleración constante. Se podría también hallar la aceleración constante de uno de los móviles, como en este ejemplo:

- En la batalla final, los caminantes blancos avanzan en línea recta al encuentro de los habitantes de Invernalia. En el instante en que ambos están separados 500 metros, los caminantes tienen una velocidad de $10 \mathrm{~m} / \mathrm{s}$ que cambia a razón de $3 \mathrm{~m} / \mathrm{s}$ cada segundo, mientras que los soldados de Invernalia tienen una velocidad inicial de $20 \mathrm{~m} / \mathrm{s}$ y una aceleración constante. Si ambos se encuentran a $225 \mathrm{~m}$ de la posición inicial de los caminantes blancos, ¿con qué aceleración se desplazan los soldados de Invernalia y cuánto tiempo tardan en encontrarse los dos ejércitos?

\subsection{Caída libre (CL)}

La serie ofrece también oportunidades para tratar la caída libre como caso particular del MRUA, comparando las escenas de la caída del rey Tommy desde la torre con la caída al "vacío" de la tía de Sansa Stark empujada por Meñique en Nido de águilas. Algunos factores a analizar serían la velocidad inicial de caída (en el primer caso nula y en el otro, no) o la influencia de la masa de la persona o del rozamiento en la caída. A partir de datos conocidos, se puede proponer calcular con qué diferencia de tiempo llegarían ambos al suelo si caen desde la misma altura o si lo harán con la misma velocidad. Es interesante hacer esquemas gráficos de los movimientos para conocer el sentido de cada variable implicada, así como resolverlo con distintos sistemas de referencia, lo que permite definir el convenio de signos más adecuado.

Otras ideas a explorar podrían ser si se mantiene la velocidad inicial a lo largo de la caída o si la persona tarda el mismo tiempo en recorrer la primera mitad del recorrido que la segunda. La caída en Nido de águilas permite introducir el concepto de vacío y discutir la ley de caída libre en la Tierra y en condiciones de vacío, introduciendo el valor de la aceleración de la gravedad a través de dicha ley: "Todos los cuerpos caen en el vacío con la misma aceleración, llamada aceleración de la gravedad".

\section{Movimiento circular uniforme (MCU) y uniformemente acelerado (MCUA)}

El estudio del movimiento circular se puede abordar a través de la escena de Drogon sobrevolando en círculos la fosa de Daznak para salvar a Daenerys de los Hijos de la Arpía. Se empezará identificando la trayectoria circular que describe el dragón y dónde interesa ubicar el sistema de referencia. Enseguida se discutirán qué magnitudes son útiles para describir este movimiento, introduciendo magnitudes angulares y su relación con magnitudes lineales. Luego, debemos plantear

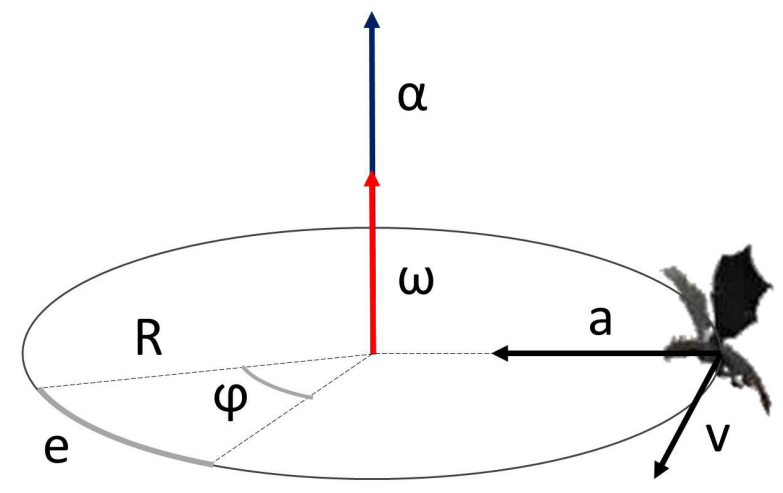

FIGURA 2. Magnitudes angulares y lineales del movimiento circular del dragón [elaboración propia].

si la velocidad con la que vuela Drogon es constante o variable con el tiempo, para poder distinguir entre un movimiento con velocidad angular $\omega$ uniforme (MCU) u otro con una velocidad angular que varía con el tiempo y, por tanto, con aceleración angular $\alpha$ constante (MCUA).

Se recomienda realizar un dibujo con el movimiento del dragón (Fig. 2) para representar sobre él la dirección y el sentido de todas las magnitudes implicadas (posición, velocidad, aceleración) tanto angulares como lineales. Para el MCU, los estudiantes pueden deducir la dirección y el sentido de la velocidad angular como el producto vectorial de la velocidad lineal por el radio de giro. El período y la frecuencia del movimiento se relacionará con el número de vueltas que da el dragón en un tiempo dado.

Se debe resaltar que, si el dragón se mueve con MCU, la velocidad angular es constante y, por ello, la aceleración angular es nula. Esto no significa que Drogon no posea ninguna aceleración, ya que tiene una aceleración lineal no nula (aceleración normal o centrípeta), que es precisamente la responsable del movimiento en círculos.

A modo de ejemplo se puede plantear este problema:

- Si Drogon vuela en círculos con una velocidad constante de $20 \mathrm{Km} / \mathrm{h}$ en un radio de $30 \mathrm{~m}$, calcular: a) La distancia que recorre en 2 segundos; b) el ángulo que se ha desplazado en ese tiempo; c) la velocidad angular del movimiento; d) la frecuencia del movimiento si tarda en dar una vuelta $8 \mathrm{~s}$.

\subsection{Tiro horizontal (TH)}

Las llamaradas de fuego lanzadas por el dragón sobre Desembarco de Rey permiten estudiar el tiro horizontal (TH) como composición de movimientos. En primer lugar, el estudiante debe identificar qué trayectoria (rectilínea, algún tipo de curva...) realizará el fuego del dragón y concluirá con la descripción de los dos tipos de movimientos que componen el TH, un MRU en el eje X y un MRUA en el eje Y. Luego, conviene realizar un esquema para identificar las magnitudes del movimiento y deducir las ecuaciones de posición, velocidad y aceleración en ambos ejes. En función de los datos que se 
propongan, esta escena permite analizar, por ejemplo, la distancia horizontal a la que llegará el fuego del dragón, tomando como referencia el punto desde el que lanzó el fuego; por otra parte, se puede analizar la velocidad con que el dragón expulsó el fuego. El enunciado de un posible problema sería:

- Si el dragón lanza una llamarada de fuego horizontalmente desde una altura de $20 \mathrm{~m}$ con una velocidad inicial de $10 \mathrm{~m} / \mathrm{s}$, ¿a qué distancia desde ese punto llegará el fuego?

\subsection{Tiro parabólico (TP)}

Varios personajes de la serie intentan matar a los dragones con diferentes estrategias. Jaime lo intentó con una gran ballesta o el Rey de la Noche lanzándole una flecha. Ambos movimientos corresponden con el lanzamiento de un cuerpo con una velocidad inicial que forma un ángulo con el eje X, donde la trayectoria descrita por el móvil corresponde a una parábola, de ahí el nombre de tiro parabólico (TP) u oblicuo. Los estudiantes deben empezar deduciendo las ecuaciones de posición, velocidad y aceleración en ambos ejes como composición de movimientos (MRU en el eje X y MRUA en el eje Y). Asimismo, deben constatar que la diferencia del TP con TH es el ángulo de lanzamiento. Es importante resaltar la altura y el alcance máximos como características del TP. Las escenas propuestas darían lugar a plantear diferentes problemas mediante lo cuales se podría calcular (a) el ángulo que debe tener la ballesta o la flecha para alcanzar al dragón, (b) la velocidad con la que debe lanzarse a cierto ángulo para impactar en el dragón, (c) la distancia a la que llegará la flecha en función del ángulo de lanzamiento o (d) la altura máxima que alcanzará. Debemos insistir en que los propios estudiantes deben proponer los valores de los datos que necesiten para su resolución, de manera que el siguiente sería un posible ejemplo:

- Si la ballesta lanza una flecha con una velocidad inicial de $100 \mathrm{~m} / \mathrm{s}$ y un ángulo de $30^{\circ}$, ¿alcanzará al dragón que se encuentra a una altura de $127.5 \mathrm{~m}$ y a una distancia de $441.5 \mathrm{~m}$ a la derecha desde donde se lanzó la flecha?

\section{Reflexiones finales}

Este trabajo ha presentado ejemplos de cómo se puede iniciar a los estudiantes en el estudio de la cinemática a través de se- cuencias de Juego de Tronos. En este sentido, cabe resaltar que la serie de televisión es la excusa para motivar a los estudiantes por el estudio de la física, de forma que logremos atraer su atención por los contenidos y producir de esta forma aprendizajes más significativos. De este modo, antes de utilizar este recurso en el aula, es interesante sondear entre el alumnado acerca de las series que están siguiendo para lograr una mayor motivación intrínseca hacia el aprendizaje.

Por otra parte, y como se ha mostrado en este trabajo, las diferentes escenas abren un campo amplio de distintos tipos de problemas a trabajar en el aula, que en todos los casos se deben plantear de forma abierta con idea de que el alumnado pueda proponer datos para su resolución, que siempre deben ser realistas.

La implementación de este tipo de tareas en el aula de secundaria revela una mejora en la percepción de la física por parte del alumnado, que indica haber aprendido más trabajando la física a través de situaciones reales y cotidianas, alejándose de esa percepción negativa inicial de que esta materia es aburrida.

Del mismo modo, una experiencia previa con la misma serie con profesores de secundaria en formación inicial en el Máster en Profesorado de Educación Secundaria de la Universidad de Málaga (España) [11] mostró la importancia de la formación en este tipo de estrategias a los futuros docentes, en particular a la hora de seleccionar las secuencias más adecuadas para aprender física y las preguntas que se deben presentar al alumnado. Tras vivenciar el recurso, los profesores en formación inicial valoraron sus posibilidades educativas con 4.54 puntos sobre 5, frente a 1.96 puntos antes de su uso. Finalmente, este trabajo pretende animar a profesores de física a utilizar las series de ficción como una estrategia didáctica que produce buenos resultados en el aprendizaje.

\section{Agradecimientos}

Este artículo forma parte del Proyecto I+D+i del Plan Nacional, referencia PID2019-105765GA-I00, titulado "Ciudadanos con pensamiento crítico: Un desafío para el profesorado en la enseñanza de las ciencias", financiado por el Ministerio de Ciencia e Innovación del Gobierno de España en la convocatoria 2019, y del Proyecto de Innovación Educativa PIE19-139 "Educación STEAM en la formación de estudiantes universitarios" financiado por la Universidad de Málaga (Málaga, España).
1. A. Vázquez A., M. A. Manassero, El declive de las actitudes hacia la ciencia de los estudiantes: un indicador inquietante para la educación científica, Revista Eureka sobre Enseñanza y Divulgación de las Ciencias, 5 (2008) 274. http://dx.doi.org/10.25267/Rev Eureka_ensen_divulg_cienc
2. J. Solbes, R. Montserrat, R. C. Furió, El desinterés del alumnado hacia el aprendizaje de la ciencia: Implicaciones en su enseñanza, Didáctica de las Ciencias Experimentales y Sociales, 21 (2007) 91.

3. Asociación para la investigación de medios de comunicación, Resumen general 2018 (AIMC, Madrid, Spain, 2018). 
4. A. Dudo, D. Brossard, J. Shanahan, D.A. Scheufele, M. Morgan, N. Signorielli, Science on Television in the 21st Century: Recent Trends in Portrayals and Their Contributions to Public Attitudes Toward Science, Communication Research, 38 (2011) 754. https://doi.org/10.1177/ 0093650210384988

5. F.J. Perales, J.M. Vílchez, The teaching of physics and cartoons: Can they be interrelated in secondary education?, Inter. J. Sci. Edu. 27 (2005) 1647. https://doi.org/10.1080/ 09500690500206366

6. A.J. Franco-Mariscal, óptica con Peppa Pig, Alambique, Didáctica de las Ciencias Experimentales, 83 (2016) 57.

7. A.J. Franco-Mariscal, Aprende física con Prison Break, Alambique, Didáctica de las Ciencias Experimentales, 60 (2009) 82.

8. S. Hughes, https: / / www. theguardian.com/

tv-and-radio/2014/mar/22/

game-of-thrones-whats-not-to-love (2014).

9. S. B. Dyson, Otherworldly Politics: The International Relations of Star Trek, Game of Thrones, and Battlestar Galactica (Johns Hopkins University Press, Baltimore, 2015).

10. R. C. Thompson, Fire, Ice, and Physics: The Science of Game of Thrones (MIT Press, Cambridge, 2019).

11. A. J. Franco-Mariscal, M. J. Cano-Iglesias, J.M. HierrezueloOsorio, Game of Thrones to learn science. An experience with Spanish pre-service science teachers, The beauty and pleasure of understanding: engaging with contemporary challenges through science education (Proceedings of ESERA 2019) (ESERA, Bologna, Italy, 2020) (in press). 